\title{
The Role of Interoperable, Agnostic and Flexibility Enabling Interfaces for DSO and System Coordination
}

\author{
Pedro Marques $^{1 *}$, João Falcão ${ }^{1}$, Susete Albuquerque ${ }^{1}$, Ricardo Bessa ${ }^{2}$, Clara Gouveia ${ }^{2}$, \\ David Rua ${ }^{2}$, José Villar ${ }^{2}$, Helena Gerard ${ }^{3}$, Kris Kessels ${ }^{3}$, Kirsten Glennung ${ }^{4}$, Antonello \\ Monti $^{5}$, José Pablo Chaves Ávila \\ ${ }^{1}$ E-REDES, Portugal \\ ${ }^{2}$ INESC-TEC, Portugal \\ ${ }^{3}$ VITO, Belgium \\ ${ }^{4}$ EDSO, Belgium \\ ${ }^{5}$ RWTH Aachen and Fraunhofer FIT, Germany \\ ${ }^{6}$ COMILLAS, Spain \\ *carlospedro.marques@e-redes.pt
}

Keywords: INTEROPERABILITY, INTERFACES, INFORMATION EXCHANGE, FLEXIBILITY, SYSTEM COORDINATION, MARKET DESIGN

\begin{abstract}
Flexibility is key for the decarbonization of the energy sector, contributing to decrease uncertainty in the operation of distribution networks, due to the connection of renewable energy sources and electric vehicles. However, effective deployment requires interoperable and replicable solutions, technologically agnostic and independent from the role of each actor and market models adopted. This paper presents an overview of ongoing projects that aim to deliver and demonstrate interoperable solutions across the full value chain of the energy sector. The main objective and expected results of the H2020 InterConnect, EUniversal and OneNet projects will be presented.
\end{abstract}

\section{Introduction}

Within the Energy Union framework, the EU aims at transforming the energy systems towards a sustainable, carbon-free, and citizen-centric economy. To enable this transformation process, DSOs face a new paradigm of operation relying increasingly on smart grids, interoperable systems and flexibility markets to safely host renewable energy sources and integrate new loads of active consumers. The increase in distributed resources combined with the needed active participation of consumers, and the transition to electric mobility and heating is triggering a key question: How do we ensure a smooth integration of flexible assets and renewable resources while maintaining an efficient, secure and reliable electricity system operation?

To address this question, new strategies, systems and tools, within the trinomial Grid-Systems-Market, have to be defined, tested and implemented to be used by SOs. The development of new activities such as the procurement of flexibility services for congestion management and nonfrequency ancillary services creates new responsibilities for the DSOs. To that end, new processes and regulatory frameworks are needed to provide proper incentives for investing in innovative solutions to ensure security of supply and cost-efficient grid management systems, with permanent alignment between SOs, FSPs and Consumers.

The increasing need to manage the electric grids to best exploit the available and alternative flexibility sources, produces different interests among SOs and Markets, and creates discussion over the topic of neutral market facilitation. On the other hand, the need to enlarge and sustain an important level of active consumer participation in the exchange of flexibility and uptake of new business models, has led to the creation of a common interest to interlink systems to attain interoperable and agnostic collaboration and coordination between SOs and other market parties.

As interoperable and replicable solutions, open standard interfaces are acknowledged as one of the keys to unlock flexibility, regardless of the role of each actor in the electric system and the ownership of solutions or platforms. This seamless mechanism should be able to ensure bidirectional provision of data and services independent of the nature of the solution, be compliant with cyber-security requirements and GDPR, and allow for direct control and supervision of each solution by the policy associated to the regulation of each member state. This way, the creation of a standard market-enabling interface linking smart grid solutions to flexibility markets 
will enhance the system coordination at national and European level and enlarge the scope for cooperation, data exchange and resources sharing between TSOs and DSOs.

This approach is being put together in the InterConnect, EUniversal and OneNet projects, with different scopes of technical development and application to safeguard the promise towards a carbon neutral Europe by 2050 .

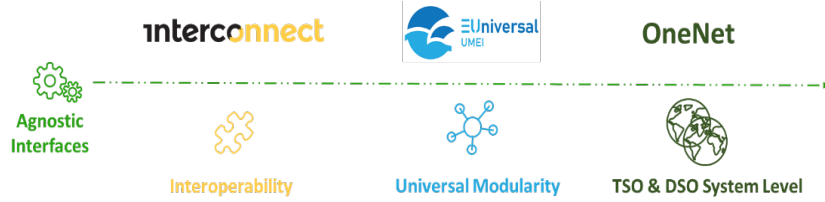

Figure 1. General overview of H2020 projects InterConnect, EUniversal and OneNet.

This paper will demonstrate the relevance of the developments of the H2020 InterConnect, EUniversal and OneNet projects. All three projects target a seamless, effective and agnostic integration of flexibility services in day-to-day operation of the future electricity system, providing specific guidelines with respect to roles, responsibilities, information and data exchange and coordination needs between parties.

\section{Review of policy and position papers}

The Clean Energy Package indicates that DSOs should procure flexibility services under transparent, nondiscriminatory and market-based procedures unless the market-based provision of these services is economically not efficient. The challenge is now how efficient marketbased procurement procedures could be arranged at the distribution level, considering the already established markets at transmission level, which, at the same time, are increasingly targeting flexibility providers connected at the distribution level. TSO, DSO and energy regulator associations have published several reports and position papers on the topic to feed the public and policy debate on the creation of an appropriate TSO-DSO-consumer framework and related market design. Several shared recommendations can be deducted, which are considered as important attention points in the three projects:

- TSOs and DSOs should clearly define their needs for flexibility to support product development and for supporting tools to solve these needs, including observability, controllability, and system operation with extended System Operator coordination [1][3];

- DSOs should get the right incentives to choose the most cost-efficient solution to solve their needs in consumers' interest, including market-based procedures, with appropriate CAPEX/OPEX consideration [1];
- Harmful interferences between DSO and TSO services should be avoided [4] and a level playing field for market parties is required, fostering new services and valuing flexibility services correctly [2];

- Roles and responsibilities, including the role of neutral market facilitator for DSOs and TSOs, should be further clarified at a European level [1][3][4];

- Non-discriminatory data management and standardisation of TSO-DSO data exchange should support efficient market functioning, including data access for TSOs related to users connected to the distribution grid [3][4];

- The resulting market framework for the procurement of DSO and TSO services can give room for development and regulation of certain aspects to fit to the national framework and situation [1], in particular regarding data management [4].

\section{Ongoing projects: a three-level approach}

This section presents an overview of ongoing projects that aim to deliver and demonstrate interoperable solutions across the full value chain of the energy sector, aiming at exploiting flexibility from DER (including demand-side resources) in scenarios characterized by high integration level of RES. The InterConnect project covers the interaction and data exchange between consumers (domestic and buildings) and DSO/third-party service providers. The EUniversal will extend the DSO interface concept, by introducing the Universal Market Enabling Interface (UMEI), a universal, open, adaptable, and modular approach to interlink active system management with electricity markets and foster the provision of flexibility services. The UMEI will support a set of novel tools for predictive management of distribution grids ( $\mathrm{LV}$, MV and HV) with flexible DER and considering resilience, while demonstrating flexibility market platforms. In the OneNet project, the Portuguese demonstrator will extend the UMEI concept to the integration between DSO and TSO, completing the multistakeholder value-chain, from TSO - DSO - Consummer.

\subsection{InterConnect: Interoperable smart homes and grids}

The InterConnect project concept is based on the following four elements: 1) foster interoperability between devices, systems and domains (e.g., domestic consumers, buildings, digital platforms from service providers, smart distribution grid) by making use of standards, ontologies and abstraction layers, as well as considering security and privacy-by-design practices; 2) design energy and nonenergy services that span across multiple levels (e.g., consumers, building, community, energy retailer) using SAREF (Smart Appliances REFerence) compliant systems and generate business value in a competitive environment; 3) design a DSO standardized interface to interact with market platforms and ensure connectivity with DSO legacy systems, enabling new business models and 
keeping a secure grid operation; 4) the replication of innovative solutions in different regions and setups allowing to move from single solutions to an integrated management at a higher scale, while focusing on scalability and replicability.

The core idea is to develop cloud-based digital marketplaces, managed and commercialized by different providers, to offer energy and non-energy services to different customers (see Fig. 2), ensuring that these services work seamlessly together (SAREF-ization of services and interoperable marketplace toolbox for platforms). This enables adopters to combine these ingredients into custom IoT based services faster and more cost effectively to generate more engagement and revenue.

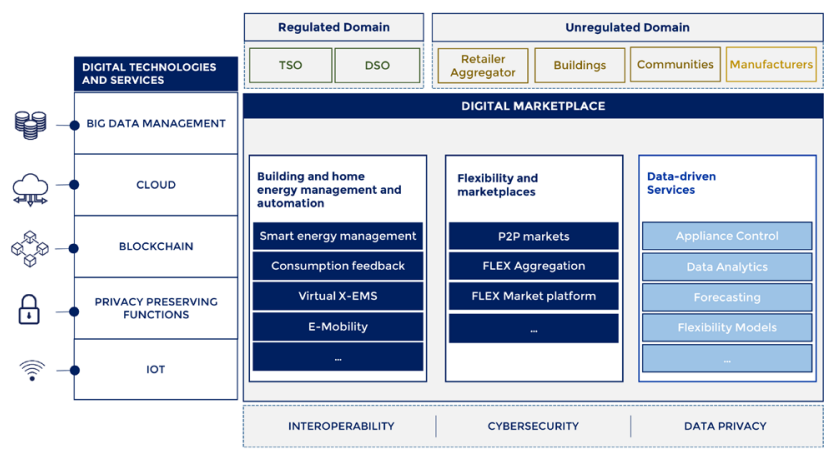

Fig. 2 InterConnect's conceptual architecture

Digital technologies (e.g., big data analytics, AI, blockchain) will enable innovative energy-related concepts, such as peer-to-peer (P2P) markets with blockchain, "virtualized" customer energy manager (CEM) for both residential (home energy management system, HEMS) and non-residential buildings (building energy management system, BEMS) and flexibility aggregation interoperable with DSO standardized interfaces. Home ecosystems, where data can be shared among the different connected devices and combined with digital technologies, will offer new non-energy services to residential customers and benefits.

Semantic interoperability based on ontologies brings a significant value proposition for Internet-of-Things (IoT) integration. IoT standards such as oneM2M, ETSI ISG CIM (NGSI-LD), EEBUS and platforms such as FIWARE have now fully embraced semantic interoperability for continuous integration in IoT, and SAREF is also reaching the maturity levels needed for commercial deployments at scale. InterConnect will use industry standards already extended using SAREF for semantic interoperability, in alignment with the joint whitepaper of AIOTI, IEEE, one $\mathrm{M} 2 \mathrm{M}$ and $\mathrm{W} 3 \mathrm{C}$, that depicts semantic interoperability in IoT principles and value propositions [5]. SAREF, SAREF4BLDG and SAREF4ENER will be analysed to understand how to enhance and adapt them to implement the InterConnect use cases. This may lead to further extensions of SAREF to cover additional domains besides energy.

\subsection{EUniversal: Flexibility \& Universal Market Enabling Interface}

The EUniversal project aims to unlock the use of distributed flexibility to address future distribution network's needs with a high integration level of RES, by integrating the use of this flexibility into the planning and operation processes of the network.

To do so, the project starts with an initial understanding, identification and characterization of the future DSO grid needs and services, adequacy of existing flexibility resources and market models and mechanisms. Table I summarizes the needs and services identified.

Table I: EUniversal DSO services

\begin{tabular}{|c|c|}
\hline Needs/Services & Definitions \\
\hline $\begin{array}{l}\text { Congestion } \\
\text { Management } \\
\text { - Corrective (real-time) } \\
\text { - Predictive (day- } \\
\text { ahead/intraday) }\end{array}$ & $\begin{array}{l}\text { Mitigate congestions caused by } \\
\text { high power consumption during } \\
\text { peak hours, concentrated charging } \\
\text { of EVs or excessive power } \\
\text { generation from DGs, among } \\
\text { other. }\end{array}$ \\
\hline $\begin{array}{l}\text { Voltage Control } \\
\text { - Corrective (real-time) } \\
\text { - Predictive (day- } \\
\text { ahead/intraday) }\end{array}$ & $\begin{array}{l}\text { Keep voltages within safe bands } \\
\text { and restore their values to normal } \\
\text { range after grid disturbances, to } \\
\text { minimise reactive power flows, } \\
\text { investments. }\end{array}$ \\
\hline $\begin{array}{l}\text { Support for network } \\
\text { planning ( } 1 \text { to } 3 \text { years } \\
\text { timeframe) } \\
\text { - Voltage control } \\
\text { (power-based) } \\
\text { - Congestion } \\
\text { management } \\
\text { (capacity based) }\end{array}$ & $\begin{array}{l}\text { Use flexibility in combination with } \\
\text { grid investments to solve either } \\
\text { current or forecasted physical } \\
\text { congestions related to reduced } \\
\text { network capacity (overload or } \\
\text { voltage violation). }\end{array}$ \\
\hline - Phase balancing & $\begin{array}{l}\text { Maintain the balance of loads } \\
\text { among phases, increase the } \\
\text { distribution network capacity, } \\
\text { reduce the risk of failures, and } \\
\text { improve voltage profiles. }\end{array}$ \\
\hline $\begin{array}{l}\text { Support for extreme } \\
\text { events } \\
\text { - Islanding } \\
\text { - Blackstart } \\
\text { - Emergency load } \\
\text { control/ Interruptible } \\
\text { load/DER } \\
\text { - Backup generation } \\
\text { capacity }\end{array}$ & $\begin{array}{l}\text { Increase the resiliency of } \\
\text { distribution networks. } \\
\text { - Islanding: in case of main grid } \\
\text { outages } \\
\text { - Blackstart: ability to restart } \\
\text { after a blackout, guaranteeing } \\
\text { grid stability and ensuring } \\
\text { adequate active and reactive } \\
\text { power balance. } \\
\text { - Emergency load control: under } \\
\text { increased demand or unplanned } \\
\text { events, provide load reductions } \\
\text { that lessen stress on the electric } \\
\text { grid. } \\
\text { - Backup generation capacity: to } \\
\text { make sustainable power } \\
\text { available for islanded operation. }\end{array}$ \\
\hline
\end{tabular}


This preliminary phase is the starting point of the EUniversal architecture based on the UMEI, that will materialize in the conceptual architecture design and the implementation of a standard, agnostic, adaptable and modular Application Programming Interface (API) to link DSOs and market parties with flexibility market platforms, in coordination with other flexibility users, see Figure 2.

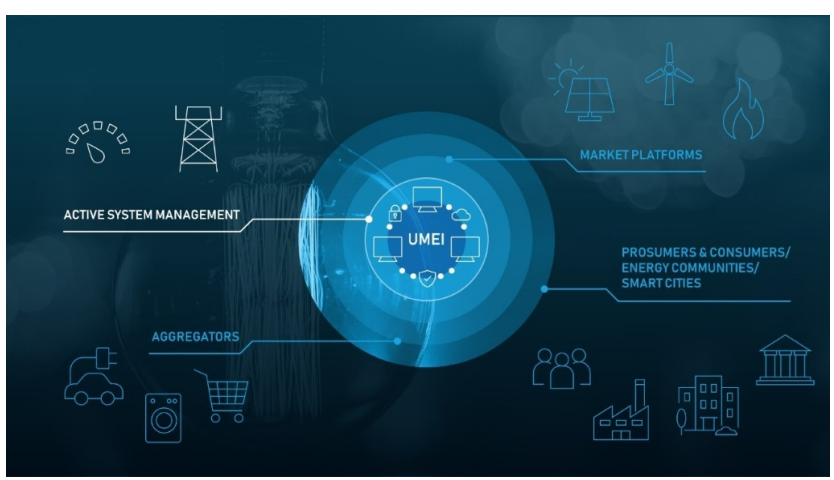

Fig. 2 EUniversal conceptual architecture

Built on top of the UMEI API, new market solutions for innovative DSO services, and integration tools for active consumer, prosumer, and energy communities' provision of flexibility for an improved and smarter distribution grid operation, will be developed.

The EUniversal project, focuses on market-based procurement of flexibility to cover the (future) DSO needs introduced above. The DSO has a variety of flexibility options and procurement mechanisms at its disposal, but not all mechanisms are equally-suited to solve the needs of the DSOs and deliver the related flexibility services. The nature of the service, the time-frame of procurement, the envisioned product requirements and the grid conditions (topology, voltage level, grid constraints) will define the most appropriate market mechanism. In the first place, explicit market mechanisms, i.e. the procurement via flexibility market platforms is considered, mainly targeted towards congestion alleviation and voltage control. The EUniversal demonstrators will test two different platforms, so that the interoperability of the platforms can be proven. The interplay with other implicit mechanisms (e.g. dynamic network tariffs, connection agreements, incentives and regulated payments) will also be explored in the EUniversal project. Furthermore, DSOs have technical solutions using grid assets at their disposal, e.g. grid reconfiguration to alter power flows, which need to be considered jointly with the market-based mechanisms. Finally, grid reinforcement will also always be an option and needs to be compared to the aforementioned marketbased flexibility options. An integrated approach is thus needed, considering all the aforementioned options and solutions.
A set of business use cases (BUC) for system services integrating the use of flexibility in new planning and operation tools will be designed and developed to be demonstrated in three different demonstrators. In order to support operation, the tools that will be developed include:

- Improve network observability. The main objective is to avoid large investments in real-time monitoring by taking advantage of historical data from grid metering and smart meter data. This includes the integration of MV load allocation and LV state estimation tools to characterize the LV network status, in terms of voltage.

- Predictive network applications: anticipating potential network restrictions, ensure optimized use of network assets, quantifying flexibility needs and validating flexibility market results both in day-ahead and intraday.

- Coordinated control framework between network voltage levels and with TSO. A multi-level optimization framework is being design in order to ensure the coordinated mobilization of flexible resources in the distribution network, complying with TSO flexibility requirements.

- Improve network operational resilience, including flexibility in operation and maintenance planning, increasing the capacity to deal with unexpected events, considering for example self-healing capabilities.

The interplay of explicit market-based flexibility acquisition mechanisms with other implicit tariff-based mechanisms will also explored in some of the EUniversal BUCs, in combination with DSOs' assets and grid reconfiguration mechanisms. For the long-term, grid reinforcements will be combined with flexibility options for the optimal grid planning.

The demonstrators will be implemented and tested in three different geographical pilots (Germany, Poland and Portugal) to prove the universality of the concept, with the aim to up-scale and replicate it beyond the life of the project and across Europe. The Portuguese demonstrator will consist of 4 different sites that will include and coordinate $\mathrm{MV}$ and $\mathrm{LV}$ resources such as residential and industrial consumers, EV chargers and storage assets. The German demonstrator will be based on a LV-grid with a relevant infeed of RES and new customers with storage and heat pump assets. The Polish demonstrator will include a local balancing area with a well automated MV grid, part of a LV grid with large shares of renewable and flexibility sources.

\subsection{OneNet: TSO-DSO Cooperation}

The OneNet framework aims to create a fully replicable and scalable architecture that enables the whole European electrical system to operate as a single system in which a 
variety of markets allows the universal participation of stakeholders regardless of their physical location, at every level from small consumers to large producers [6].

The project will combine a cost-effective open agnostic architecture based on the concept of an interoperable network of platforms with near real-time multi-country coordinated operation of a variety of innovative market models and harmonized services/products where new technologies such as edge cloud technology supported by blockchain mechanisms, provide an easy, secure and geographically universal access to customer level data for operation and flexibility markets.

OneNet integrates contributions from other H2020 projects, including EUniversal and:

- CoordiNet and Interrface that paved the way to defining the schemas for TSO-DSO cooperation

- EUSysFlex that defined key services for flexibility

- Platone that complements EUniversal at DSO level focusing on the operational level and platform architecture

The Portuguese demonstrator will extend the UMEI concept to the cooperation between DSO and TSO, completing the multi-stakeholder value-chain, from TSO DSO - Consumer. The following main ideas, departing from [7], will be further developed:

- Design, detail and implement the mechanisms for the bidirectional exchange of information between DSO and TSO, and between them and market parties, to ensure the smooth implementation of active system management requirements, regardless of the market arrangements and based on open and universal guidelines and solutions (e.g., UMEI).

- Extend the bidirectional exchange of forecasting, planning and operational information between DSO and TSO to improve coordinated system operation taking into account the toolbox of possible flexibility solutions.

- Improve the estimation of the potential of consumer flexibility provision on the basis of statistics, supported by large and representative consumer segments.

\section{Conclusion}

This paper provides a complementary overview of three H2020 projects InterConnect, EUniversal and OneNet projects, regarding its objectives and main outcomes, considering the common vision for a seamless, effective and agnostic integration of flexibility services in the operation of the future electricity system, providing specific guidelines with respect to roles, responsibilities, information and data exchange and coordination needs between parties.

All three projects present a coherent and incremental roadmap to the integration of market-based flexibility services into the DSO planning and operation.

\section{Acknowledgements}

This work has been funded by European Union's Horizon 2020 research and innovation programme under grant agreements No 857237, 864334 and 957739 InterConnect, EUniversal and OneNet projects

\section{References}

[1] CEER., 'CEER Paper on DSO Procedures of Procurement of Flexibility' (July 2020), Ref: C19-DS-55-05.

[2] CEDEC, EDSO, ENTSO-E, Eurelectric, GEODE, 'TSO-DSO Report An integrated approach to active system management' (2019).

[3] CEDEC, EDSO, ENTSO-E, Eurelectric, GEODE, 'TSO-DSO Data Management Report' (2016).

[4] EDSO., 'Facilitating customers energy data management and interoperability -DSOs' perspective' (November 2020).

[5] Bassbouss, L., Telekom, A. D., Bauer, M. N., et al., 'Semantic interoperability for the web of things', ResearchGate Online resource, 2016, 19 $\mathrm{pp}$.

[6] Monti, A., 'Definition of a global architecture for smart grid applications' Working Document, 2018. [Online] https://ec.europa.eu/newsroom/dae/document.cfm ?doc_id=54669

[7] Brazier, R., Cunha, L., Hermans, P., de Jong, G., Knop, T., Lallemand, M., et al., 'An integrated approach to active system management with focus on TSO-DSO coordination in congestion management and balancing', Technical Report, 2019, pp. 1-4 